\section{Angiotensin receptor blockers (ARBs)}

Guertin and colleagues describe potential savings from restricting use of ARBs in Canada. ${ }^{1}$ The US Department of Veterans Affairs prefers angiotensinconverting-enzyme (ACE) inhibitors over ARBs, with national criteria for use to guide prescribing.

Even in a closed system such as Veterans Affairs, however, there are opportunities for savings; we find substantial variation in the use of ARBs and ACE inhibitors across the system. For 2009, we examined variations among facilities in the proportion of patients taking a renin-angiotensin system blocker who were also taking an ARB. ${ }^{2}$ The median proportion was $17.2 \%$ (lower than the $25 \%$ described by Guertin and colleagues for 2006) but ranged across facilities from $6.4 \%$ to $33.6 \%$. In an analysis in fiscal year 2010, we found that if all regions in Veterans Affairs were to use ARBs at a rate not higher than the national average, the savings to the department could be US $\$ 2.4$ million per year (unpublished data). If all facilities were to use ARBs at a rate not higher than the lowest-using five facilities, the savings would have been US\$6.5 million per year.

We concur with the authors that careful evaluation of patterns of drug use can identify opportunities for substantial cost savings, where patient outcomes are not likely to be adversely affected. Such variation in precribing patterns suggests that factors beyond patient variability affect drug choice. Even small differences in patterns of use can have large cost implications at a national level, as the authors demonstrated for just one drug class in Canada.

\section{Walid F. Gellad MD MPH}

Centre for Health Equity Research and Promotion, VA Pittsburgh Healthcare System, Assistant professor of medicine, University of Pittsburgh, Pittsburgh, Penn.

\section{Bernie Good MD MPH}

VA Pittsburgh Health Care System, University of Pittsburgh School of Medicine, Pittsburgh, Penn.

\section{References}

1. Guertin JR, Jackevicius CA, Cox JL, et al. The potential economic impact of restricted access to angiotensin-receptor blockers. CMAJ 2011;183: E180-6.

2. Gellad WF, Donohue J, Good CB, et al. Use of angiotensin receptor blockers in the VA, 20002009: Why so much variation? J Gen Intern 2010; 25(Suppl 3):S421.

CMAJ 2011. DOI:10.1503/cmaj.111-2036

\section{Malnutrition, age and in- hospital mortality}

We recently showed that malnutrition is independently associated with a prolonged length of hospital stay. ${ }^{1}$

In a further analysis of our data, we investigated the association of malnutrition (nutritional risk index $<97.5$ ) and age ( $\leq 65$ years v. 65 years) with in-hospital mortality by a conditional logistic regression model stratified by diagnosis of cancer and hospital stay in a medical or surgical ward.

Older patients $(44.4 \%)$ were more frequently at nutritional risk than younger patients $(39.7 \%$ v. $49.7 \%, p<$ $0.001)$. The in-hospital mortality rate was $4.1 \%$ ( $n=52 ; 95 \%$ confidence interval $[\mathrm{CI}] 3.0-5.2)$. The rate was $1.5 \%$ (95\% CI 0.7-2.7) in patients with normal nutritional status and $7.1 \%(95 \%$ CI 5.1-9.4) in malnourished patients.

The crude odds ratio (OR) for inhospital death was 4.27 (95\% CI 2.09$8.75, p<0.001)$. There was a significant interaction between age and nutritional risk $(p=0.023)$, with age identified as a strong effect modifier. Indeed, in patients aged 65 years or less, mortality was $0.5 \%$ (95\% CI $0.0-1.7)$ in the absence of malnutrition and $7.0 \%$ (95\% CI 4.4-10.3) if malnutrition was present. The OR for death was 11.91 (95\% CI 2.74-51.79), $p<0.001)$. Conversely, in patients aged more than 65 years, the difference in mortality was much lower $(3.0 \%, 95 \%$ CI $1.3-5.7$ v. $7.1 \%, 95 \%$ CI 2.5-10.2), and the OR for death was 2.19 (95\% CI 0.91-5.24, $p=0.07$ ).

Our data emphasize the importance of nutritional screening at the time of hospital admission, because malnutrition could represent a strong predictor of mortality, especially in nonelderly patients.
Further multivariable studies with adequate sample sizes are needed to investigate the association among malnutrition, age and in-hospital mortality.

\section{Riccardo Caccialanza MD \\ Emanuele Cereda MD PhD \\ Nutrition and Dietetics Service \\ Catherine Klersy MD \\ Biometry and Clinical Epidemiology \\ Service \\ Istituto di Ricovero e Cura a Carattere Scientifico, Fondazione Policlinico San Matteo, Pavia, Italy}

\section{Reference}

1. Caccialanza R, Klersy C, Cereda E, et al. Nutritional parameters associated with prolonged hospital stay among ambulatory adult patients. CMAJ 2010;182:1843-9.

CMAJ 2011. DOI:10.1503/cmaj.111-2038

\section{Depression: addressing our blind spots}

Serious medical conditions may be missed because of the presence of a psychiatric history. ${ }^{1}$ Most of us who work at the interface of medicine and psychiatry would agree with the authors that such a consequence is not intentional.

In the system's efforts to reduce wait times, attempts are made to find efficiencies wherever possible. This may include making assumptions based on probabilities - for example, that most chest pain is noncardiogenic and that a psychiatric history might make this even more likely.

However, the death rate for people with mental illness is about $70 \%$ higher than that for the rest of the population. As the complexity and age of patients continues to rise, we need to ensure that proper medical care is provided to everyone, including those with a history of psychiatric illness. This is very important in emergency departments with parallel process models where a patient with a psychiatric history can be initially seen by someone in psychiatry instead of the emergency physician.

Patients with psychiatric illnesses will only be assured the best of care by: - educating practitioners in emer- 